\title{
28 Research Square \\ Quantitative Analysis of Hypervascular Hepatic Metastases and Hepatocellular Carcinoma using Spectral CT Imaging
}

\section{Zhiqiang Yang}

Shandong Qianfoshan Hospital https://orcid.org/0000-0002-7779-4625

\section{Xinyi Wang}

Shandong Provincial Qianfoshan Hospital, Shandong University

Hao Shi (D19937062@qq.com)

https://orcid.org/0000-0001-5334-0795

\section{Research article}

Keywords: Hepatic metastasis, Hypervascularity, Hepatocellular carcinoma (HCC), Spectral imaging, Tomography, X-ray computer, Quantitative analysis

Posted Date: November 18th, 2019

DOl: https://doi.org/10.21203/rs.2.17420/v1

License: (c) (i) This work is licensed under a Creative Commons Attribution 4.0 International License. Read Full License 


\section{Abstract}

Objective The goal of this study is to evaluate the performance of spectral CT-based quantitative analysis in differential diagnosis of hypervascular hepatic metastasis (HVHM) and hepatocellular carcinoma (HCC).

Methods Spectral CT scans were performed for 47 patients with hepatic malignant tumors, including 20 patients with HVHM and 27 patients with HCC, which generated the following sets of data: single energy images in the arterial phase; iodine and water maps; marginal areas of lesions that manifested apparent signal intensification; and energy spectral parameters of normal liver tissues and abdominal aorta. Subsequently, we calculated the normalized iodine concentrations (NIC), lesion-normal parenchyma iodine concentration ratio (LNR), iodine concentration difference (ICD) between the arterial phase and the venous phase, and the spectral curve slope. An independent samples t test and receiver operating characteristic (ROC) curve analysis were applied to examine these quantitative parameters.

Results In the arterial phase, the HVHM and HCC groups displayed no differences in NIC, LNR, or spectral curve slope $(P>0.05)$. In the venous phase, the two groups displayed significant differences in NIC, LNR, and spectral curve slope; the NIC was $0.59 \pm 0.08$ for the HVHM group and $0.45 \pm 0.10$ for the HCC group; the LNR was $1.17 \pm 0.22$ and $0.92 \pm 0.16$, respectively; the spectral curve slope was $1.85 \pm 0.49$ and 1.18 \pm 0.34 , respectively. In addition, there was no significant difference in ICD between the HVHM group (0.54 $\pm 0.39 \mathrm{~g} / \mathrm{L})$ and $\mathrm{HCC}$ group $(0.45 \pm 0.39 \mathrm{~g} / \mathrm{L})(P>0.05)$. Finally, there were no significant differences of water or iodine concentration between the arterial phase and venous phase $(P>0.05)$. Taken together, the spectral curve slope in the portal venous phase had the best performance in differentiating HVHM from HCC.

Conclusions HVHM and HCC have apparent differences in spectral curve and concentrations of radiocontrast agents in the portal venous phase. Hence, spectral CT imaging provides a new multiparameter quantitative approach for differentiating HVHM and HCC.

\section{Background}

Based on the degree of blood supply, malignant liver tumors can be divided into hypervascular lesions and hypovascular lesions. Common malignant hypervascular tumors in the liver include hepatocellular carcinoma (HCC) as well as hepatic metastases that originate from gastrointestinal mucinous adenocarcinoma, gastrointestinal stromal tumor, renal cell carcinoma, breast cancer, sarcoma, choriocarcinoma or other sources (1). Hypervascular hepatic metastasis (HVHM) and HCC differ considerably in tumorigenesis, biological behavior, and prognosis. Hence, reliable differentiation between $\mathrm{HVHM}$ and $\mathrm{HCC}$ is crucial for accurate staging, treatment plan formulation, and prognosis assessment. The development of spectral imaging computed tomography (CT) provides a new means for the diagnosis and identification of hepatic space-occupying lesions. Spectral imaging overcomes the singleparameter problem of traditional CT imaging and generates multiparametric images for the quantitative 
analysis of lesions. In this study, quantitative parameters from spectral CT was analyzed retrospectively to differentiate the lesions of HVHM and HCC, in which the different energy spectral parameters were compared. Based on the results, we evaluated the performance of spectral imaging CT-based quantitative analysis in the differential diagnosis of HVHM and HCC.

\section{Materials And Methods}

Clinical information Between October 2013 and September 2015, 47 patients with malignant hepatic tumors underwent enhanced CT scanning in Qianfoshan Hospital, Shandong University. The cohort included 32 males and 15 females, had an age range of 33-78 years, a median age of 57 years, and contained 20 patients with HVHM and 27 patients with HCC. The size range of HVHM and HCC were 5.1$13.6 \mathrm{~cm}$ and $6.6-15.3 \mathrm{~cm}$ respectively, a median size of $8.2 \mathrm{~cm}$ and $9.6 \mathrm{~cm}$. The primary tumors of the HVHM patients included colorectal cancer $(n=5)$, gastric cancer $(n=3)$, gastrointestinal stromal tumor $(n$ $=2)$, melanoma $(n=1)$, kidney cancer $(n=4)$, and breast cancer $(n=5)$. The inclusion criteria of the HVHM group (2-3) were as follows: both primary tumors and liver metastases were confirmed with operation and biopsy pathology; enhanced CT scan revealed signs consistent with apparent arterial phase manifestations including ring-like, nodular, or diffuse intensification. The inclusion criteria of the HCC group were as follows: all patients were confirmed with operation and biopsy pathology; enhanced CT scans revealed pronounced intensification in the arterial phase and decreased intensification in the portal venous phase. This study was approved by the Medical Ethics Committee of the hospital.

\subsection{Methods}

1.2.1 Scanning methods CT was performed using a GE spectral CT scanner (Discovery 750 HDCT; GE Healthcare, Milwaukee, Wis). This spectral CT has achieved 4 technological breakthroughs: i) a highperformance gemstone detector; ii) spectral imaging and coronary subtraction angiography; iii) 500 slices 4-dimensional dynamic coverage; iv) high definition and less dose image. The GSI that we used in our study could momentary switch between high and low energies ( $140 \mathrm{kV}$ and $80 \mathrm{kV})$ in a single X-ray tube in 0.5 milliseconds. This makes material differentiation with monochromatic energy feasible. The monochromatic imaging can help eliminate the ray-hardening artifacts, optimize imaging quality, and increase image contrast. 9 With GSI, we can get 101 monochromatic images from $40 \mathrm{keV}$ to $140 \mathrm{keV}$ and derive images for the separation of materials such as calcium, iodine, and water. These provide the theoretical basis for our analysis. All patients underwent abdominal enhanced scanning under gemstone spectral imaging (GSI) mode. Fast switching of tube voltages allowed instant transition between low and high energy states ( $80 \mathrm{kVp} / 140 \mathrm{kVp}$ ); the tube current was approximately $550 \mathrm{Ma} / \mathrm{r}$; the rotation speed of $\mathrm{X}$-ray tube was $0.5 \mathrm{~s} / \mathrm{r}$; the pitch was 0.985 ; the detector width was $0.625 \mathrm{~mm} \times 64$; the slice thickness was $5 \mathrm{~mm}$; and the scan range was from top of diaphragm to lower edge of liver. Contrast agent iodohexanol (dose of $1.2 \mathrm{~mL} / \mathrm{kg}$, concentration of $300 \mathrm{mg} / \mathrm{mL}$ ) was injected into peripheral veins with a high-pressure syringe at a flow rate of $3.0 \mathrm{~mL} / \mathrm{s}$. Prior to the arterial phase scan, the abdominal aortic CT 
value was used to monitor and trigger scanning with a monitoring threshold of $120 \mathrm{HU}$; after reaching the threshold, the scanning was delayed for 8 seconds before being initiated. The scan was initiated 28 seconds after the end of the arterial phase.

1.2.2 Image postprocessing The original data of the arterial and portal vein phases were reconstructed to generate images with a slice thickness of $0.625 \mathrm{~mm}$, which were imported into an Aw4.4 workstation and analyzed using a GSI viewer. lodine-based images, waterbased images, and 101 sets of monochromatic images were obtained first. Then iodine concentration, water concentration, spectral curve, and CT values at $40 \mathrm{keV}$ in the ROI of the leision were measured with GSI postprocessing software. In the HVHM group, there were a total of 179 lesions, including 6 solitary lesions in 6 subjects. In the HCC group, there were 43 lesions, including 21 solitary lesions. For each participant, only one typical lesion with good image quality and no fusion was selected for measurement, in which a circular or oval region of interest (ROI) was placed in a highly enhanced area on the tumor edge, avoiding zones of necrosis, large vessels, calcification and sclerotic artifacts. The parameters that required measurement and calculation included the following: i) iodine (water) concentration and water (iodine) concentration in the lesions, and iodine concentration in normal liver tissue and abdominal aorta; ii) NIC = iodine concentration of lesion $\left(\mathrm{IC}_{\text {lesion }}\right)$ /iodine concentration of abdominal aorta $\left(\mathrm{IC}_{\text {abdominal aorta }}\right)$; iii) $\mathrm{LNR}=\mathrm{IC}_{\text {lesion }}$ /iodine concentration of normal liver tissue $\left(\mathrm{IC}_{\text {liver }}\right)$; iv) ICD = [iodine concentration in the arterial phase $\left(I \mathrm{C}_{\text {arterial phase }}\right)$ ]-[iodine concentration in the portal venous phase $\left.\left(\mathrm{IC}_{\text {portal venous phase }}\right)\right]$; spectral curve slope $=(\mathrm{CT} 50 \mathrm{keV}$ CT100keV)/(100keV-50keV). Two radiologists with 4 and 9 years of experience in abdominal CT interpretation, respectively, evaluated the images. In cases of discordant interpretations, decisions were reached by consensus. All data were measured in triplicate, and the average value was used in the analysis.

Statistical analysis SPSS 17.0 was used for statistical analyses. Independent samples t-test was performed to compare NIC, LNR, ICD, iodine concentrations, and spectral curve slope in the arterial and portal venous phases between the HVHM group and HCC group. The resulting data were expressed as \pm s. The diagnostic efficacy of each quantitative parameter to differentiate the HVHM group and HCC group was assessed by a ROC curve. $P<0.05$ was considered statistically significant.

\section{Results}

1.1 Comparison of spectral quantitative parameters Typical manifestations of HVHM and HCC in arterial phase and portal venous phase, as well as spectral parameters were displayed in Figure 1 and Figure 2 respectively. The HVHM group and HCC group exhibited no significant differences in NIC, LNR, and spectral curve slope of the arterial phase $(P>0.05)$ but had significant differences in these parameters of the portal vein phase $(P<0.01)$. In addition, the two groups displayed no difference in iodine concentration of the arterial or portal venous phase $(P>0.05)$ or ICD $(P>0.05)$. The results are summarized in Table 1. 
1.2 Diagnostic efficacy evaluation of spectral quantitative parameters for HVHM and HCC Analysis of Receiver Operating Characteristic (ROC) curves was performed to examine whether the spectral quantitative parameters were capable of differentiating the HVHM group and HCC group. The results (Table 2) revealed that the spectral curve slope in the portal venous phase had the largest area under the ROC curve (AUC), indicating the best diagnostic performance; when spectral curve slope was greater than 1.420 , the sensitivity and specificity for HVHM were $90 \%$ and $88.9 \%$, respectively. When the NIC was greater than 0.555 in the portal venous phase, the sensitivity and specificity for HVHM were $75 \%$ and $85.2 \%$, respectively. In comparison, the LNR in the portal venous phase showed the lowest efficacy in differentiating the two groups; when the LNR was greater than 0.955 , the sensitivity and specificity for HVHM were $80 \%$ and $88.9 \%$, respectively.

\section{Discussion}

Because the liver receives dual blood supply from the hepatic artery and portal vein, extrahepatic malignant tumors can easily metastasize to the liver, resulting in a relatively high incidence of liver metastasis. It has been reported that most liver metastases are hypovascular lesions. Conventional contrast-enhanced CT has high sensitivity and specificity in the diagnosis of liver metastasis. On the other hand, HVHM is relatively rare and has an incidence of $4 \%-7 \%$, but its manifestations in enhanced CT are drastically different from those of common hypovascular metastases. Specifically, HVHM often manifests pronounced ring-like, nodular, and diffuse intensification in the arterial phase, but the degree of intensification gradually decreases in the portal venous phase and the delayed phase (4). Therefore, images of HVHM are often similar to those of HCC (5), which is especially the case for patients with extrahepatic primary malignant tumors who have no reliable disease history or have solitary lesions. Hence, conventional contrast-enhanced CT alone has limitations in differentiation between HVHM and $\mathrm{HCC}$ and can easily lead to misdiagnosis.

Spectral CT imaging is based on instantaneous switching $(0.5 \mathrm{~ms})$ between the high and low energy states, which can not only obtain images of various substrate materials (e.g., water-based, iodine-based, and calcium-based maps) via material separation technology but also generate spectral curves of the lesions. Different parameters reflect different spectral characteristics. The degree of blood supply can be indirectly revealed by detecting the iodine concentration in tissues (6). A spectral curve can reveal the Xray attenuation coefficients, which correspond to the variations of different pathological tissues in response to the change of a single energy level (7). Differences between spectral curves can be expressed by slope, which provides a basis of quantitative analysis to distinguish different pathological types of tumors. It has been reported that quantitative analysis based on spectral CT imaging has made a breakthrough in the diagnosis, differentiation, and prognosis evaluation of different types of tumors (7-9).

The iodine map in spectral CT imaging is particularly sensitive to iodine deposition. The measurement of iodine concentration in the iodine map can directly reveal intratumor iodine distribution, which is indicative of the blood supply. The results of this study showed that in the arterial phase, both HVHM and HCC tumors manifested obvious enhancement and had little differences in blood supply, iodine 
concentration, and NIC. Most of the HCC patients had concurrent liver cirrhosis, which was characterized by extensive proliferation of hepatic fibrous connective tissue and formation of intrahepatic pseudolobules. These lesions cause the obstruction of portal vein circulation, resulting in the decrease of portal venous perfusion and the compensatory increase of hepatic arterial blood supply. Therefore, in the arterial phase, the iodine concentration of normal liver tissue in HCC patients is increased, whereas LNR is decreased. Of note, tumors in both HVHM and HCC participants were verified to be hypervascular lesions. It was reported that the DSA manifestations of HVHM were often similar to those of HCC; both types of lesions were easily stained in the late arterial phase and mainly manifested as thickening, twisting, disorganization of hepatic artery, venous lakes, and arteriovenous fistulas (9). Studies have shown that the spectral parameters in the portal venous phase have important clinical values in differentiating the benign and malignant liver lesions and that iodine concentration analysis of spectral CT imaging can be used to differentiate small hepatocellular carcinoma from small hemangioma; in particular, iodine concentration analysis in the portal venous phase had a sensitivity and specificity of $97 \%$ and $100 \%$ for differentiating the two types of tumors (10-11). Our results showed that in the portal venous phase, the HVHM group had spectral parameters for NIC and LNR significantly higher than those of the HCC group, indicating that the contrast-enhancement degree of the HVHM group was higher than that of the HCC group, which might be attributed to the blood supply and pathological structure of the two types of tumors. It is widely accepted that HVHM tumors are mainly supplied by the hepatic artery, followed by the portal vein, which is referred as dual blood supply. Using an animal model, Voboril et al. (12) demonstrated that the portal vein can supply blood directly or indirectly to the margin of metastatic tumors through hepatic sinuses. Infiltration of inflammatory cells from the tumor edge or increased release of endothelial growth factors resulted in continuous contrast-enhancement of HVHM tumors in the portal phase (13). In addition, in the portal venous phase, contrast agents withdraw from the HVHM and HCC tumors. However, CT perfusion imaging showed that the contrast agent exhibited short residence time and fast clearance in HCC but slow dispersion in HVHM (14-15). As a consequence, the HVHM lesions showed a slow reduction in enhancement degree in the portal venous phase, indicating that in this period, the iodine concentration of the HVHM group was lower than that of the HCC group. Our results show that the parameters based on iodine concentration can quantitatively reveal the degree of blood supply of HVHM and HCC lesions. Although it is not easy to differentiate the two types of tumor in the arterial phase, the iodine concentration in the portal venous phase can provide more reliable information to differentiate the two. Nevertheless, the HVHM and HCC groups exhibited no significant difference in water (iodine) concentration in dual phase scanning, indicating that this parameter has no diagnostic value in differentiating HVHM and HCC.

It was reported that different pathological types of human tissues can be distinguished by spectral curves (16). Our results revealed that the spectral curve slope in the portal venous phase was significantly higher in the HVHP group than the HCC group. In fact, the spectral curve slope in the portal venous phase displayed the best performance in differentiating these two types of liver tumors. HVHM and HCC are different in pathological types, tumor growth and metabolism, and vascularity, which is reflected by the difference in their CT values on the spectral curves. The difference is more pronounced at the low keV 
single energy level, when the spectral curves of HVHM and HCC differ greatly, leading to relatively high sensitivity and specificity in their differentiation. It was shown that HCC displayed a higher spectral curve slope than liver metastases (17), which was contrary to our findings and might be related to the blood supply types of the selected metastases. Of note, the liver metastases examined by Wang et al. (17) were hypovascular tumors originated from pancreatic cancer, esophageal carcinoma, and lung cancer. In contrast, hypervascular tumors were examined in this study. Our analyses revealed that in the arterial phase, both HVHM and HCC display apparent enhancement and have little difference in blood supply, having a minimal effect on the attenuation of CT values. As a consequence, the spectral curves in the arterial phase have poor diagnostic performance. Our data also suggest that mechanistically, the spectral curve is similar to the iodine concentration. The spectral curve indicates that the lower the single energy level, the greater the CT value; the greater the reduction of CT values, the greater the spectral curve slope. At the same time, the higher the iodine concentration is, the greater the CT value as well as the spectral curve slope. In other words, increased iodine concentration is associated with increased blood supply and spectral curve slope. As such, the slope of spectral curve is a robust quantitative index in differentiating HVHM and HCC because the slope of spectral curve can not only reveal the different pathological types of tumor but also quantify the different degrees of vascularity of tumors and amplify the difference. Correspondingly, the spectral curve slope can provide valuable information for quantitatively determining tumor origin and thus is a promising quantitative marker for differentiating HVHM and HCC.

There are some deficiencies in this study. For example, the selection of the ROI is particularly important. The accurate selection of the ROI can obtain the accurate measurement data and calculate the accurate $\mathrm{NIC,LNR}$ and so on. In practice, sometimes it is difficult to select ROI, and the small change of its position may lead to the deviation of the measured data, which will affect the accuracy and repeatability of the test results. It often takes more time to get a more stable result. How to place the ROI accurately will be a key point in the next step.

In conclusion, spectral CT imaging displays good capacity in differentiating HVHM and HCC, which is based on quantitative analysis of the NIC, LNR and spectral curve slope. In particular, the spectral slope curve in the portal venous phase generates relatively high sensitivity and specificity, which is helpful to improve the accuracy of differential diagnosis and provide a basis for the formulation of a treatment plan and an assessment of prognosis.

The main points

1, Spectral CT imaging is a kind of functional imaging, which can not only obtain images of various substrate materials (e.g., water-based, iodine-based, and calcium-based maps) via material separation technology but also generate spectral curves of the lesions. The above parameters can be used to evaluate the lesion in a new angle.

2, The iodine map in spectral CT imaging is particularly sensitive to iodine deposition. The measurement of iodine concentration in the iodine map can directly reveal intratumor iodine distribution, which is indicative of the blood supply. 
3 , In different pathological types of tissues, there are significant differences in their spectral curves. The spectral curve slope can provide valuable information for quantitatively determining tumor origin.

\section{References}

[1] Namasivayam S, Salman K, Mittal P, et al. Hypervascular hepatic focal lesions: spectrum of imaging features [J]. Curr Probl Diagn Radiol, 2007, 36(3):107-123.

[2] Zhou K. Chinese imaging medicine: Chapter of liver, bile, pancreas, and spleen [M]. Beijing: People's Medical Publishing House (PMPH), 2002:67.

[3] Christine S, Stephen T, Ivancevic MK, et al. Diffusion-weighted MRI of metastatic liver lesions: is there a difference between hypervascular and hypovascular metastases? [J]. Acta Radiol, 2014, 55(5):515-523.

[4] Matos AP, Altun E, Ramalho M, et al. An overview of imaging techniques for liver metastases management [J]. Expert Rev Gastroenterol Hepatol, 2015, 9(12):1561-1576.

[5] Bhayana D, Kim TK, Jang HJ, et al. Hypervascular liver masses on contrast-enhanced ultrasound: the importance of washout [J]. AJR Am J Roentgenol, 2010, 194(4):977-983.

[6] Chae EJ, Song JW, Krauss B, et al. Clinical utility of dual-energy CT in the evaluation of solitary pulmonary nodules: initial experience [J]. Radiology, 2008, 249(2):671-681.

[7] Liu J, Liu Y, Li L et al. Preliminary study of spectral CT imaging in the differential diagnosis of metastatic lymphadenopathy due to various tumors [J]. Chinese Journal of Radiology, 2011,45(8):731735.

[8] Paul J, Vogl TJ, Mbalisike EC. Oncological applications of dual-energy computed tomography imaging [J]. J Comput Assist Tomogr, 2014, 38(6):834-842.

[9] Chen Z, Zhang Z, Zhao X, et al. Angiographic analysis of 269 cases of liver metastases [J]. Jilin Medical Journal, 2010, 31(25):4360-4361.

[10] Lv P, Lin XZ, Li J, et al. Differentiation of small hepatic hemangioma from small hepatocellular carcinoma: recently introduced spectral CT method [J]. Radiology, 2011, 259(3):720-729.

[11] Yu Y, He N, Sun K, et al. Differentiating hepatocellular carcinoma from angiomyolipoma of the liver with CT spectral imaging: a preliminary study [J]. Clin Radiol, 2013, 68(9):491-497.

[12] Voboril R. Blood supply of metastatic liver tumors: an experimental study [J]. Int Surg, 2005, 90(2):7177. 
[13] Semelka RC, Hussain SM, Marcos HB, et al. Perilesional enhancement of hepatic metastases: correlation between MR imaging and histopathologic findings-initial observations [J]. Radiology, 2000, 215(1):89-94.

[14] Hayano K, Desai GS, Kambadakone AR, et al. Quantitative characterization of hepatocellular carcinoma and metastatic liver tumor by CT perfusion [J]. Cancer Imaging, 2013, 13(4):512-519.

[15] Xue M, Bai R, Li F et al. Differential value of CT perfusion imaging in hepatocellular carcinomas, hepatic metastastes and hemoangiomas. [J]. International Journal of Medical Radiology, 2008, 31(3): 152-155.

[16] Wei W, Yu Y, Lv W, et al. Predictive value of dual-energy spectral computed tomographic imaging on the histological origin of carcinomas in the ampullary region [J]. Abdom Imaging, 2014, 39(4):702-710.

[17] Wang Q, Shi G, Qi X, et al. Quantitative analysis of the dual-energy CT virtual spectral curve for focal liver lesions characterization [J]. Eur J Radiol, 2014, 83(10):1759-1764.

\section{Declarations}

Ethics approval and consent to participate区

The experimental protocol was established, according to the ethical guidelines of the Helsinki Declaration and was approved by the Human Ethics Committee of Shandong Provincial Qianfoshan Hospital, Shandong University. Written informed consent was obtained from individual or guardian participants.

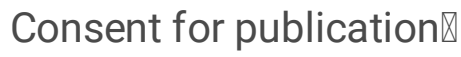

Not applicable.

Competing interests $\mathbb{Z}$

The authors declare that they have no competing interests"

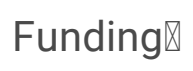

This study was supported in part by grants from the science and technology program of Shandong Province (2011GSF11828) 
Authors' contributions $\rrbracket$

Yang Zhiqiang- data acquisition, data analysis and interpretation, manuscript preparation, manuscript editing

Wang Xinyi- statistical analysis, manuscript editing

Shi Hao- study concepts, study design, manuscript review

\section{Tables}

Due to technical limitations, tables are only available as a download in the supplemental files section

\section{Figures}
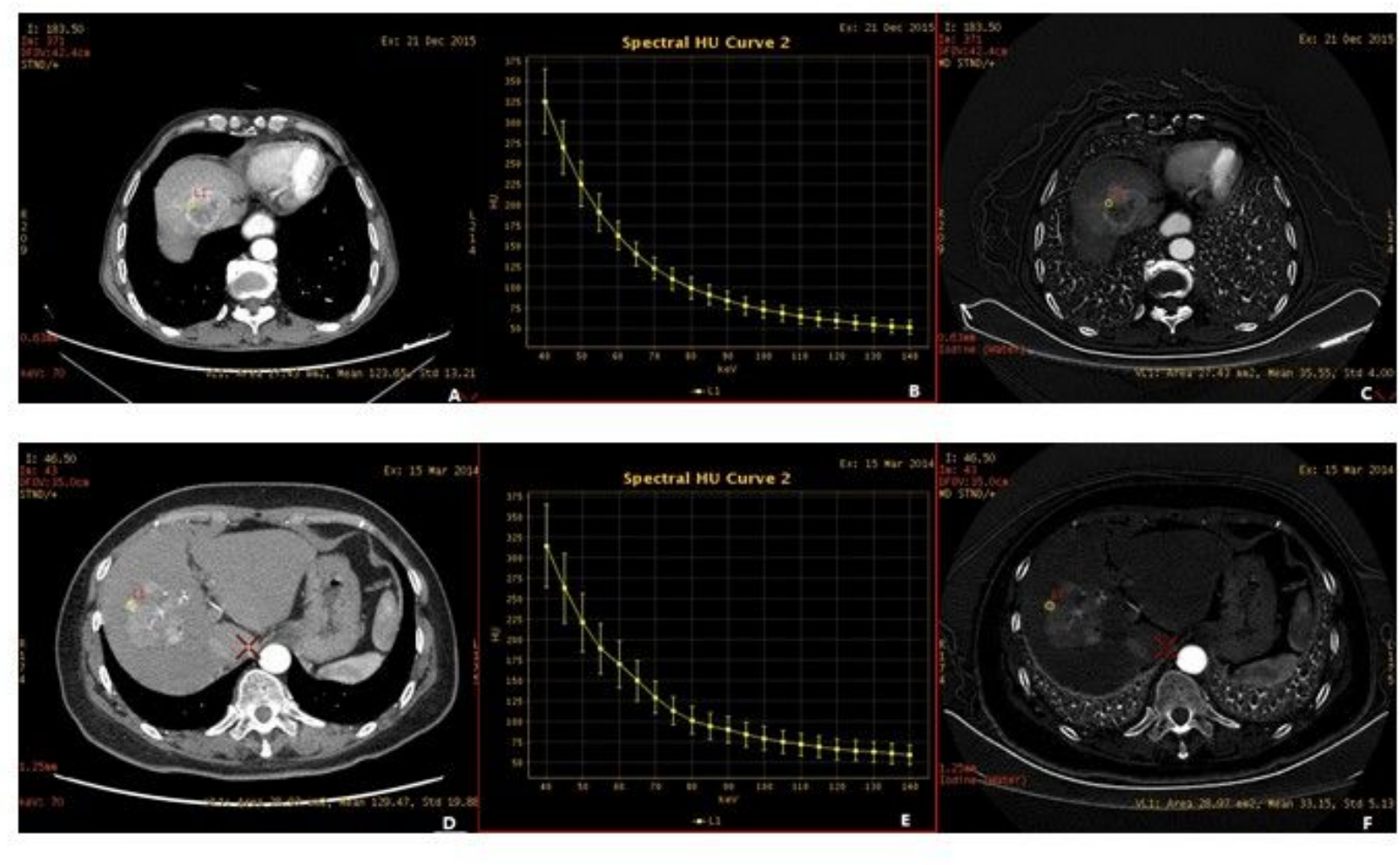

\section{Figure 1}

Spectral parameters of the HVHM group and HCC group in the arterial phases On $70 \mathrm{keV}$ monochromatic images of arterial phase, both HVHM and HCC showed obvious heterogeneous enhancement, in which the enhancement of HVHM (A) was mainly located in the peripheral region, while the enhancement of HCC (D) was heterogeneous in the whole lesion. The small yellow circle represents the region of interest 
(ROI). The corresponding spectral curve are B and E. Figure $\mathrm{C}$ and $\mathrm{F}$ were iodine map of HVHM and HCC respectively. The number shown in the lower left corner represents the iodine concentration.
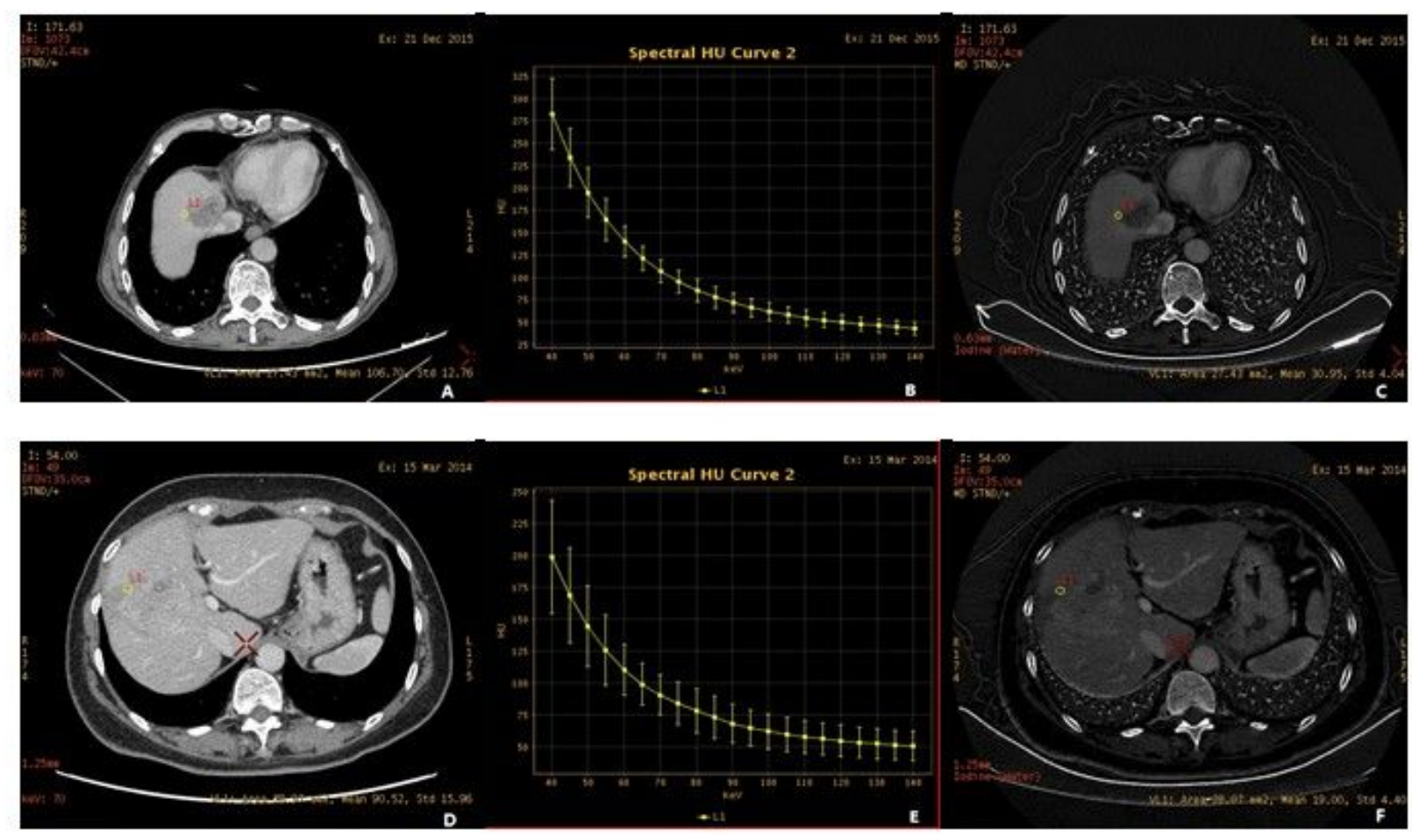

\section{Figure 2}

Spectral parameters of the HVHM and HCC groups in the portal venous phases On $70 \mathrm{keV}$ monochromatic images of portal venous phases, HVHM (A) showed mild peripheral enhancement, while HCC (D) showed decreased overall enhancement. The small yellow circle represents the region of interest (ROI). The corresponding spectral curve are B and E. Figure $\mathrm{C}$ and $\mathrm{F}$ were iodine map of HVHM and HCC respectively. The number shown in the lower left corner represents the iodine concentration. 